\title{
Prospective external validation of the Predicting Out-of-OFfice Blood Pressure (PROOF-BP) strategy for triaging ambulatory monitoring in the diagnosis and management of hypertension: observational cohort study
}

\author{
(c) (1) () \\ OPEN ACCESS
}

In this paper by Sheppard and colleagues (BMJ 2018;361, doi:10.1136/bmj.k2478, published 27 June 2018) the authors would like to acknowledge that author F D Richard Hobbs is supported by the National Institute for Health Research (NIHR) Oxford Biomedical Research Centre. The views expressed are those of the authors and not necessarily those of the National Health Service, the NIHR, or the Department of Health and Social Care. This work uses data provided by patients and collected by the NHS as part of their care and support and would not have been possible without access to this data. The NIHR recognises and values the role of patient data, securely accessed and stored, both in underpinning and leading to improvements in research and care. F D Richard Hobbs is also professor at the NIHR Oxford Biomedical Research Centre, Oxford University Hospitals NHS Foundation Trust. 